\title{
A diagnosis of knee osteoarthritis does not predict physical activity 2 years later in older adults: findings from the Hertfordshire Cohort Study
}

\author{
Michael A. Clynes ${ }^{1}$ (D) . Camille Parsons ${ }^{1} \mathbb{D} \cdot$ Mark H. Edwards $^{1,3} \mathbb{D}$. Jonathan H. Tobias ${ }^{2} \mathbb{D} \cdot$ Kevin Deere $^{2} \mathbb{D}$. \\ Cyrus Cooper $^{1,4,5}$ (D) Elaine M. Dennison ${ }^{1}$ (D)
}

Received: 12 March 2019 / Accepted: 16 April 2019 / Published online: 29 April 2019

(c) The Author(s) 2019

\begin{abstract}
Osteoarthritis (OA) can negatively impact levels of physical activity (PA), although current clinical advice promotes the benefits of staying active in preventing joint degeneration. In this study, we examine how knee OA, assessed by self-report, clinical assessment and radiographic assessment, impacts upon objectively measured PA 2 years later. The study population is comprised of 114 subjects from the Hertfordshire Cohort Study (HCS). The presence of OA at the knee was determined from self-report, and clinical and radiological examination, defined according to American College of Rheumatology (ACR) criteria and Kellgren and Lawrence grading system. Two years later, Gulf Coast Data Concepts (GCDC) tri-axial accelerometers were used to measure day-to-day levels of PA. Vertical acceleration peaks over 7 days, expressed in $\mathrm{g}$ units, were categorised into low $(0.5 \leq \mathrm{g}<1.0)$, medium $(1.0 \leq \mathrm{g}<1.5)$ and high $(\geq 1.5 \mathrm{~g})$ impacts. The study comprises 69 men and 45 women. The mean (SD) age was 78.5 (2.6) for men and 78.6 (2.7) for women. Low count numbers were recorded in the medium and high impact bands. We found no significant reduction in low, medium or high impacts in individuals who had been previously diagnosed with self-reported, radiographic or clinical knee OA in this sample after adjustment for age, sex and BMI. In our cohort, participants with knee OA were no less likely to partake in objectively measured weight-bearing activity 2 years after assessment than counterparts without a diagnosis of knee OA.
\end{abstract}

Keywords Osteoarthritis · Physical activity $\cdot$ Accelerometer $\cdot$ Knee

Michael A Clynes and Camille Parsons are joint first authors.

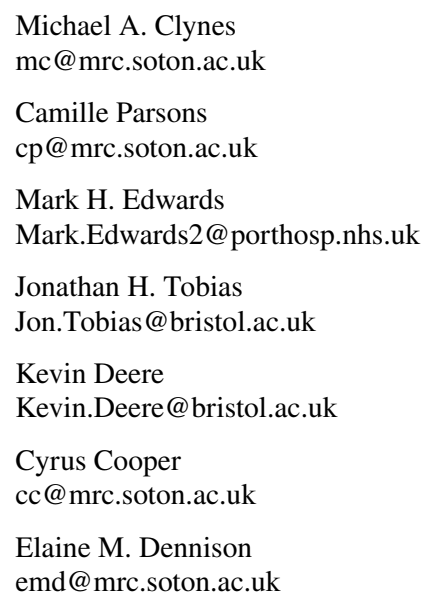

emd@mrc.soton.ac.uk

1 MRC Lifecourse Epidemiology Unit, University of Southampton, Southampton General Hospital, Southampton, UK

2 Musculoskeletal Research Unit, Translational Health Sciences, Bristol Medical School, University of Bristol, Bristol, UK

3 Portsmouth Hospitals NHS Trust, Portsmouth, UK

4 National Institute for Health Research Biomedical Research Centre, University of Southampton and University Hospital Southampton NHS Foundation Trust, Southampton, UK

5 National Institute for Health Research Musculoskeletal Biomedical Research Unit, University of Oxford, Oxford, UK 


\section{Introduction}

Of the joint disorders affecting older people, osteoarthritis (OA) is the most common. Indeed, in England and Wales it is estimated that 1.6-3.4 million people are afflicted with OA and the societal burden is significant with up to 1.9 million disabled by their symptoms [1]. In OA, there is degeneration of the joint involving the articular cartilage in addition to many of the surrounding tissues [2]. This occurs when there is a disruption of the balance between the breakdown and repair of joint tissue, which precipitates the loss of articular cartilage, remodelling of subchondral bone, osteophyte formation, ligament laxity, periarticular muscle weakening, and occasionally synovitis [3]. Individuals with OA may suffer joint pain, which in turn leads to stiffness and restricted movement. The most commonly affected joints in OA are the hands, feet, facet joints and large weight-bearing joints such as the knees and hips [2].

OA can negatively impact levels of physical activity (PA), although current advice promotes the benefits of staying active in preventing joint degeneration [4]. Epidemiological studies have traditionally relied upon the use of health questionnaires, thus not providing an objective measure of PA as brief, non-volitional periods of activity will likely be excluded [5]. Accelerometer-based PA readings have been developed as part of the Vertical Impacts on Bone in the Elderly (VIBE) study (a novel method for evaluating day-today vertical impacts from weight-bearing PA, subsequently classified according to impact magnitude) [6]. Vertical accelerations reflect impacts resulting from weight-bearing activity to which the skeleton preferentially responds. These methods have previously been used to demonstrate a positive association between levels of higher, but not medium- or low-, impact PA and lower limb bone strength in adolescents and older women [7, 8]. Applying this approach to older individuals is, however, challenging as high-impact activities such as running and jumping are rarely undertaken in this age-group. The objective of this study was to assess the feasibility of the methodology in older adults, and explore if PA may be reduced in those with a clinical, self-reported or radiological diagnosis of knee OA. We hypothesised that pain associated with self-reported or clinical knee OA may be associated with a greater effect on PA levels then radiographic knee OA which can be asymptomatic.

\section{Methods}

The participants in this study were from the Hertfordshire Cohort Study (HCS). The HCS was originally incepted to study the relationship between early life factors and risk of common adult diseases later in life. The study population for this study is comprised of 69 men and 45 women from the HCS, who were also part of the VIBE study. The methods of this study and cohort construction are described in detail elsewhere [9], but in brief we traced men and women born between 1931 and 1939 in Hertfordshire and who still lived there in 1998-2004 when a nurse-administered questionnaire and clinic visit were carried out. Participants were not selected on the basis of musculoskeletal pathology, but represented individuals born in a geographic region who continued to live there. In 2011-2012, 592 men and women from the geographical area of East Hertfordshire were invited to take part in a study that was designed to consider the personal burden of OA (European Project on Osteoarthritis (EPOSA) study [10]). In the HCS cohort, only participants who were previously included in the UK arm of the EPOSA study were invited to participate, as this group had available information relating to knee OA status. The cohort included individuals with and without a diagnosis of knee OA. 222 men and 222 women were approached and those that agreed to participate had their PA levels monitored through the use of accelerometers at follow-up. At baseline, subjects were visited at home by a trained research nurse where questionnaires were administered and clinical examinations were performed. Anterior-posterior (AP) and lateral knee radiographs were taken of both knees at a local hospital after the home visit. A self-reported diagnosis of knee OA was obtained by asking study participants "Do you have knee osteoarthritis?" and if the response was "yes" the joint affected by OA was ascertained. A total of 69 men and 45 women completed home visits and attended for X-rays.

Clinical knee OA was defined based on algorithms developed by the American College of Rheumatology [11]. A clinical diagnosis of knee OA was made if a study participant reported pain in the knee (as evaluated by the Western Ontario and McMaster Universities Arthritis Index (WOMAC) pain subscale), plus any three of: (1) bony tenderness in at least one side on examination; (2) crepitus on active motion in at least one side on examination; (3) less than 30 min of morning stiffness, evaluated by the WOMAC stiffness subscale; (4) no palpable warmth of synovium in both knees on examination; (5) age over 50 years; or (6) bony enlargement in at least one side on examination. The WOMAC is a 24-item questionnaire with three subscales measuring pain (5 items), stiffness ( 2 items), and physical function (17 items) [12].

Radiographs were graded according to Kellgren and Lawrence (KL) by two experienced rheumatologists. The KL grading system is briefly described as follows: grade 1 - unlikely narrowing of the joint space and possible osteophytes on the radiograph; grade 2 - small osteophytes and 
possible narrowing of the joint space; grade 3-multiple, moderately sized osteophytes, definite joint space narrowing, some sclerotic areas and possible deformation of bone ends; and grade 4-multiple large osteophytes, severe joint space narrowing, marked sclerosis and definite bony end deformity [13]. Here, a positive definition of radiological patellofemoral OA reflected a KL score of 2 or above in the patellofemoral joint; radiological tibiofemoral OA a KL score of 2 or above in the tibiofemoral joint; and radiological knee OA a KL score of 2 or above in either the tibiofemoral joint or the patellofemoral joint. If either knee was affected, the subject was classified as having knee OA.

Approximately 2 years after radiographs were taken, Gulf Coast Data Concepts (GCDC) X15-1c triaxial accelerometers (Gulf Coast Data Concepts, Waveland, Mississippi) with custom designed size-specific elasticated belts, worn at the hip site for 7 days, were used to measure day-to-day levels of PA as part of the VIBE study in participants who consented. Whilst wearing the accelerometer, individuals also completed a daily time log, detailing when the accelerometer was worn. Participants were instructed to position the accelerometer over their right hip pointing centrally and to remove it only for washing, swimming or sleeping. Accelerometers had a sampling frequency of $50 \mathrm{~Hz}$. The process of analysing the data retrieved from the accelerometers used a custom code and is described in detail by Deere and colleagues [14]. In summary, the raw data from the accelerometers were imported into Stata 13 (StataCorp, College Station, TX). The data were cleaned to remove non-wear time and any movement artefacts. A day of recording was excluded if it is comprised of less than $10 \mathrm{~h}$ of valid recording time. Only those with $\geq 3$ valid recording days were included in the analysis. Data were normalised based on 7 valid days ( $\geq 10 \mathrm{~h}$ recording time) of $14 \mathrm{~h}$. Vertical acceleration peaks over 7 days, expressed in $\mathrm{g}$ units, were categorised into low $(0.5 \leq \mathrm{g}<1.0)$, medium $(1.0 \leq \mathrm{g}<1.5)$ and high $(\geq 1.5 \mathrm{~g})$ impacts. Acceleration peaks were calculated based on accelerations higher than the preceding and subsequent reading. All $g$ values represent $g$ over and above $1 \mathrm{~g}$ from earth's gravitational force.

The UK component of EPOSA had ethical approval from the Hertfordshire Research Ethics Committee, reference number 10/h0311/59 on the 21/01/2014, and all participants gave written, informed consent.

\section{Statistical analysis}

Participant demographic data and questionnaire responses were presented using means and standard deviations for continuous variables and the number and percentages for categorical variables. All continuous variables were visually inspected for normality. Median and inter-quartile range (IQR) were used to summarise PA intensity counts due to the skewed nature of the counts. Comparison in counts between lower limb OA statuses was made using Wilcoxon rank-sum. Associations between lower limb OA and PA intensity was assessed using linear regression after the accelerometry data were log transformed, and a count of 1 was added to medium and high activity counts to enable a log transformation to be completed and allow adjustment for confounders (age, sex and BMI).

\section{Results}

A diagnosis of OA and useable PA activity data were available for 114 participants (69 men and 45 women). The mean (SD) age was 78.5 (2.6) for men and $78.6(2.7)$ for women. Men were taller and heavier than women $(p<0.01)$ although BMI (body mass index) was comparable. Thirteen men (18.8\%) and 8 women (17.8\%) self-reported knee OA. Similar proportions of men and women had a clinical diagnosis of knee OA, 7 (10.5\%) and 5 (11.4\%) respectively. Radiographic OA was more common than self-report or clinical OA with $41.0 \%$ of men and $34.9 \%$ of women having radiographic $\mathrm{OA}(\mathrm{KL} \geq 2)$ at the tibiofemoral joint, $32.8 \%$ of men and $37.2 \%$ of women having radiographic $\mathrm{OA}$ at the patellofemoral joint and $52.5 \%$ of men and $48.8 \%$ of women having radiographic knee OA (OA at either the patellofemoral joint or tibiofemoral joint). Only one participant had a score of KL grade 4 at the tibiofemoral joint (none at the patellofemoral joint).

Many more activity counts were recorded in the lowthan the medium-intensity range regardless of OA status (the median number of counts in these brackets ranged between 5033 and 7286 in the low-intensity range and 119-269 in the medium-intensity range) and very few counts were recorded in the high intensity band (median number of counts in this bracket ranged between 23 and 46) (Table 1).

Table 1 and Fig. 1 show relationships between PA and OA by the definitions used. Study participants with OA at the knee did not record significant differences in the median number of low, medium and high counts recorded regardless of which definition of OA used except for a significant negative association between radiographic patellofemoral OA and the number of high intensity counts ((Median (IQR) patellofemoral OA present: 23 (6-58), patellofemoral OA not present: $46(13-122) p=0.04)$ (Table 1). We then performed linear regression between OA and activity with adjustment for age, sex and BMI and the association between radiographic patellofemoral OA and the number of high intensity counts was attenuated (Fig. 1). 
Table 1 Intensity count of activity peaks by OA status

\begin{tabular}{|c|c|c|c|c|c|c|}
\hline & \multicolumn{2}{|l|}{ Yes } & \multicolumn{3}{|c|}{ No } & \multirow[t]{2}{*}{$p$ value } \\
\hline & Median & $\mathrm{IQR}^{\mathrm{a}}$ & $N$ & Median & $\mathrm{IQR}^{\mathrm{a}}$ & \\
\hline \multicolumn{7}{|c|}{ Low intensity $(\geq 0.5 \mathrm{~g}-1.0 \mathrm{~g})$} \\
\hline Clinical knee OA & 7009 & $3916-10,887$ & & 5880 & $2373-13,278$ & 0.77 \\
\hline Self-reported knee OA & 5033 & $1849-8820$ & & 6407 & $2712-13,964$ & 0.16 \\
\hline KL tibiofemoral OA & 5035 & $2159-11,363$ & & 6599 & $2880-12,852$ & 0.30 \\
\hline KL patellofemoral OA & 5044 & $1300-9543$ & & 6812 & $2756-14,802$ & 0.16 \\
\hline KL knee OA & 5037 & $2401-10,230$ & & 7826 & $2712-14,142$ & 0.18 \\
\hline \multicolumn{7}{|c|}{ Medium intensity $(\geq 1.0 \mathrm{~g}-1.5 \mathrm{~g})$} \\
\hline Clinical knee OA & 168 & $87-291$ & & 213 & $64-763$ & 0.68 \\
\hline Self-reported knee OA & 166 & $64-263$ & & 227 & $65-763$ & 0.27 \\
\hline KL tibiofemoral OA & 141 & $53-476$ & & 232 & $68-612$ & 0.26 \\
\hline KL patellofemoral OA & 119 & $53-318$ & & 232 & $76-802$ & 0.09 \\
\hline KL knee OA & 136 & $55-437$ & & 269 & $70-816$ & 0.09 \\
\hline \multicolumn{7}{|l|}{ High intensity $(>=1.5 \mathrm{~g})$} \\
\hline Clinical knee OA & 37 & $22-107$ & & 38 & $13-112$ & 0.77 \\
\hline Self-reported knee OA & 38 & $22-65$ & & 38 & $11-122$ & 0.97 \\
\hline KL tibiofemoral OA & 29 & 10-99 & & 39 & 13-105 & 0.69 \\
\hline KL patellofemoral OA & 23 & $6-58$ & & 46 & $13-122$ & 0.04 \\
\hline KL knee OA & 26 & $9-86$ & & 45 & $13-121$ & 0.19 \\
\hline
\end{tabular}

${ }^{a} \mathrm{IQR}$ inter-quartile range

Fig. 1 Relationships between log-transformed activity counts and $\mathrm{OA}$ status, according to linear regression. Black = unadjusted. Grey $=$ adjusted for age, sex and BMI

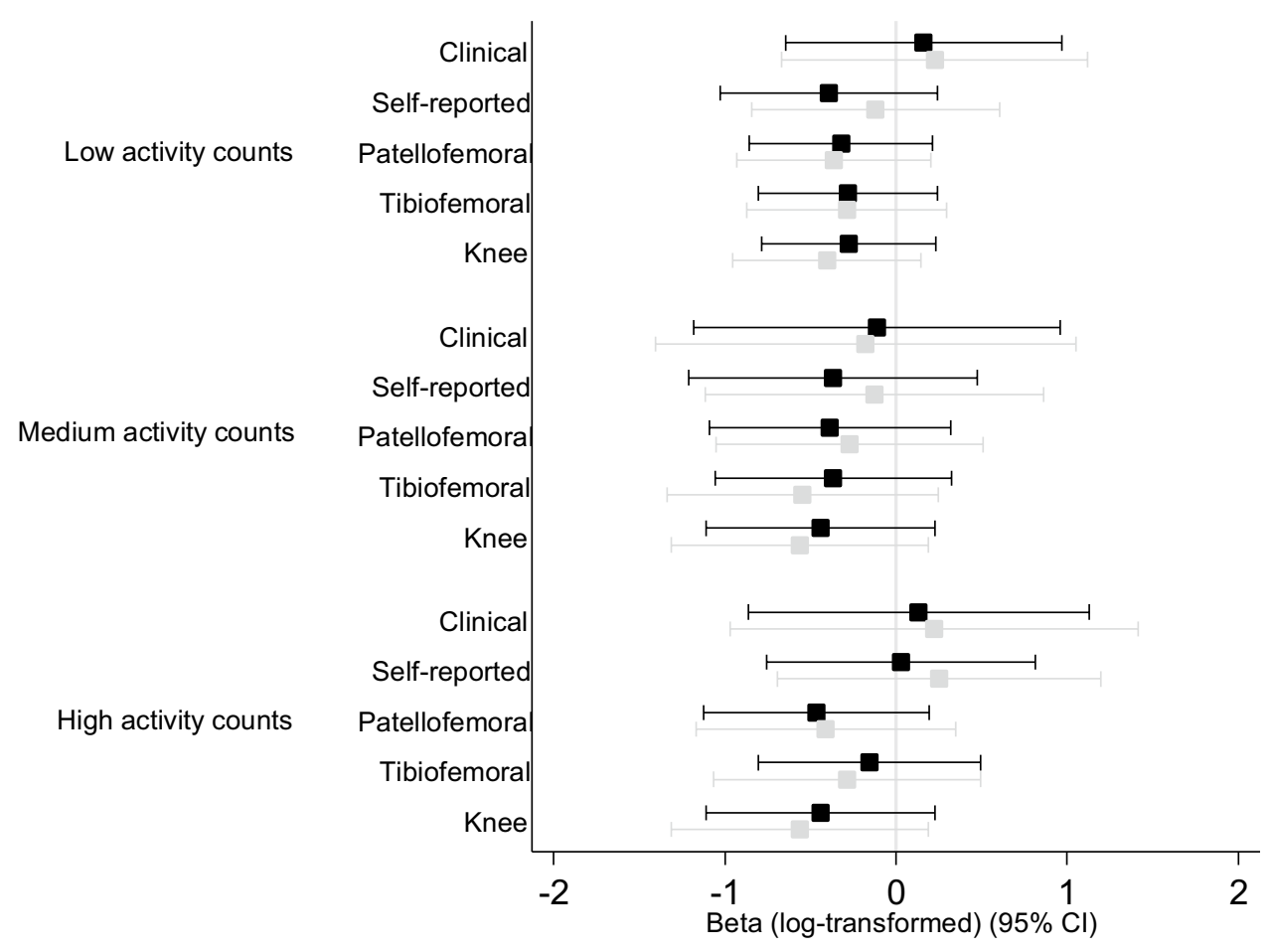

Black $=$ unadjusted

Grey $=$ adjusted for age, sex and BMI 


\section{Discussion}

We have examined the relationship between self-reported, clinical and radiographic diagnoses of knee OA and subsequent objectively assessed PA in a cohort of older adults through the use of accelerometers which recorded vertical movements and classified them based on level of impact. This study proved the methodology is acceptable to older adults who were able to wear an accelerometer for the required period of time and observed no significant relationships between any structural or symptomatic assessment of knee OA and subsequent PA after adjustment for confounders. Radiographic knee OA was more common than self-report or clinical knee OA which is consistent with previous studies [15, 16].

Since radiographic changes are known to predict symptoms poorly [17], it could be expected that a difference in PA may be observed in the subgroup with clinical OA. There did appear to be lower activity counts in the middle impact band in those participants with clinical OA but this did not reach statistical significance. Our sample was, however, too small to test this reliably and further studies on larger cohorts where clinical OA is more prevalent are warranted.

Current management of knee OA highlights the need to remain physically active despite the diagnosis. Indeed, non-surgical and non-pharmacological interventions are considered first-line treatments for knee OA as they are safe, low-cost, low-tech, incorporate self-management performed at home or in the community and have a substantial public health impact [18-20]. The uptake of guidance for individuals with knee OA to remain physically active and adherence to this has been reported in few studies. A recent questionnaire-based study by Zhou and colleagues showed that in a population of 1069 individuals in China with knee OA $93.6 \%$ of the patients thought that they could adhere to the exercise treatment if they received professional advice [21]. Other studies have shown, however, that despite exercise providing immediate and shortterm clinically worthwhile benefits, adherence to exercise declines significantly over time [22]. Our data suggest that the clinical guidelines encouraging maintenance of PA is possibly being heeded but further studies with a larger number of participants with clinical OA are needed. This study is novel because it is a longitudinal follow-up that includes an objective measure of physical activity that might be adopted in other epidemiology studies as the methodology has been shown to be feasible in an older, unselected age-group.

This study has limitations and strengths. There were only a moderate number of participants (114), limiting our power to detect statistically significant relationships and these findings would, therefore, warrant confirmation in other larger datasets. This is especially true for clinical $\mathrm{OA}$ as only 12 participants met the criteria for this and this exploratory analysis needs to be confirmed in larger data sets. Since all the participants recruited were born in the county of Hertfordshire and had continued to reside there until they were 75 , the results may not be entirely representative of the wider UK population [23]. It is also conceivable that there may be an element of selection bias, whereby healthier and more physically active individuals are more likely to partake in studies such as this one. The HCS has, however, been previously demonstrated to be a good representation of the general population with regard to body build and lifestyle factors, such as smoking and alcohol intake, therefore, suggesting that any selection bias would be small [24]. Two experienced rheumatologists were used to grade the radiographs, with high interobserver concordance and have previously shown good levels of agreement exists between- and within-observer variations [25]. In this analysis, we considered someone to have clinical/radiological knee OA if at least one knee was affected but due to low numbers were unable to consider a dose effect if both knees or other joints were affected and since only one subject had KL grade 4 our analysis included individuals with only moderate radiographic change. We do not have data on whether a participant underwent joint replacement surgery between their baseline assessments and follow-up which could increase their ability to perform weight-bearing activity.

In conclusion our results suggest that individuals with evidence of knee OA, however, classified (structural or symptomatic), were no less likely to partake in habitual weight-bearing activity 2 years after assessment than counterparts without a diagnosis of knee OA. Our study shows that objective measurement of PA in individuals with OA was feasible in this age-group and further studies in larger cohorts are now indicated.

Author contributions $\mathrm{MC}$ wrote and revised the paper and together with $\mathrm{CP}$ interpreted the data and formulated conclusions. $\mathrm{CP}$ also wrote the statistical analysis plan, cleaned and analysed the data, and revised each draft. ME was involved with study design and recruitment. JT and KD were involved with study design and reviewed the drafted manuscript. CC and ED provided supervision for the whole project, were involved with study design and recruitment, data interpretation, and revision of the drafted manuscript. All authors approved the final version.

Funding This study was funded by the Porticus foundation.

\section{Compliance with ethical standards}

Conflict of interest Author Michael Clynes has received support for attending conferences from UCB, Pfizer and Eli Lilly. Author Mark 
Edwards has received support for attending conferences from Eli Lilly, Pfizer and UCB and support for attending educational courses from Chugai and Abbvie. Author Elaine Dennison has received fees from Pfizer and UCB. Author Professor Cyrus Cooper has received lecture fees and honoraria from Amgen, Danone, Eli Lilly, GSK, Medtronic, Merck, Nestlé, Novartis, Pfizer, Roche, Servier, Shire, Takeda and UCB outside of the submitted work. Authors Camille Parsons, Kevin Deere and Jonathan Tobias have nothing to disclose.

Ethical approval All procedures performed in studies involving human participants were in accordance with the ethical standards of the national research committee and with the 1964 Helsinki declaration and its later amendments or comparable ethical standards.

Informed consent Informed consent was obtained from all individual participants included in the study.

Congress abstract publications This work was presented as a poster at Rheumatology 2017, Birmingham, UK.

Open Access This article is distributed under the terms of the Creative Commons Attribution 4.0 International License (http://creativeco mmons.org/licenses/by/4.0/), which permits unrestricted use, distribution, and reproduction in any medium, provided you give appropriate credit to the original author(s) and the source, provide a link to the Creative Commons license, and indicate if changes were made.

\section{References}

1. Clynes MA, Parsons C, Edwards MH, Jameson KA, Harvey NC, Sayer AA, Cooper C, Dennison EM (2014) Further evidence of the developmental origins of osteoarthritis: results from the Hertfordshire Cohort Study. J Dev Origins Health Dis 5(6):453-458. https://doi.org/10.1017/s2040174414000373

2. Litwic A, Edwards MH, Dennison EM, Cooper C (2013) Epidemiology and burden of osteoarthritis. Br Med Bull 105:185-199. https://doi.org/10.1093/bmb/lds038

3. Hutton CW (1989) Osteoarthritis: the cause not result of joint failure? Ann Rheum Dis 48(11):958-961

4. Brosseau L, Wells GA, Pugh AG, Smith CA, Rahman P, Alvarez Gallardo IC, Toupin-April K, Loew L, De Angelis G, Cavallo S, Taki J, Marcotte R, Fransen M, Hernandez-Molina G, Kenny GP, Regnaux JP, Lefevre-Colau MM, Brooks S, Laferriere L, McLean L, Longchamp G (2016) Ottawa Panel evidence-based clinical practice guidelines for therapeutic exercise in the management of hip osteoarthritis. Clin Rehabilitation 30(10):935-946. https://doi. org/10.1177/0269215515606198

5. Clynes MA, Wyawahare P, Robinson G, Denison HJ, Evans G, Gilmour M, Dennison EM (2015) Bone health among premenopausal female alcoholics: a pilot study. Open Bone J 7:14-18. https://doi.org/10.2174/1876525401507010014

6. Hannam K, Deere KC, Hartley A, Clark EM, Coulson J, Ireland A, Moss C, Edwards MH, Dennison E, Gaysin T, Cooper R, Wong A, McPhee JS, Cooper C, Kuh D, Tobias JH (2017) A novel accelerometer-based method to describe day-to-day exposure to potentially osteogenic vertical impacts in older adults: findings from a multi-cohort study. Osteoporos Int 28(3):1001-1011. https://doi. org/10.1007/s00198-016-3810-5

7. Hannam K, Deere KC, Hartley A, Al-Sari UA, Clark EM, Fraser WD, Tobias JH (2016) Habitual levels of higher, but not medium or low, impact physical activity are positively related to lower limb bone strength in older women: findings from a population-based study using accelerometers to classify impact magnitude. Osteoporos Int 28(10):2813-2822. https://doi.org/10.1007/s0019 8-016-3863-5

8. Deere K, Sayers A, Rittweger J, Tobias JH (2012) A crosssectional study of the relationship between cortical bone and high-impact activity in young adult males and females. J Clin Endocrinol Metab 97(10):3734-3743. https://doi.org/10.1210/ jc. 2012-1752

9. Syddall HE, Aihie Sayer A, Dennison EM, Martin HJ, Barker DJ, Cooper C (2005) Cohort profile: the Hertfordshire cohort study. Int J Epidemiol 34(6):1234-1242. https://doi.org/10.1093/ije/ dyi127

10. Schaap LA, Peeters GM, Dennison EM, Zambon S, Nikolaus T, Sanchez-Martinez M, Musacchio E, van Schoor NM, Deeg DJ (2011) European project on OSteoArthritis (EPOSA): methodological challenges in harmonization of existing data from five European population-based cohorts on aging. BMC Musculoskelet Dis 12:272. https://doi.org/10.1186/1471-2474-12-272

11. Altman RD (1991) Classification of disease: osteoarthritis. Semin Arthritis Rheum 20(6 Suppl 2):40-47

12. Bellamy N (2002) WOMAC: a 20-year experiential review of a patient-centered self-reported health status questionnaire. J Rheumatol 29(12):2473-2476

13. Kellgren JH, Lawrence JS (1957) Radiological assessment of rheumatoid arthritis. Ann Rheum Dis 16(4):485-493

14. Deere KC, Hannam K, Coulson J, Ireland A, McPhee JS, Moss C, Edwards MH, Dennison E, Cooper C, Sayers A, Lipperts M, Grimm B, Tobias JH (2016) Quantifying habitual levels of physical activity according to impact in older people: accelerometry protocol for the vibe study. J Aging Phys Activity 24(2):290-295. https://doi.org/10.1123/japa.2015-0066

15. Felson DT, McAlindon TE, Anderson JJ, Naimark A, Weissman BW, Aliabadi P, Evans S, Levy D, LaValley MP (1997) Defining radiographic osteoarthritis for the whole knee. Osteoarthr Cartil 5(4):241-250

16. Parsons C, Clynes M, Syddall H, Jagannath D, Litwic A, van der Pas S, Cooper C, Dennison EM, Edwards MH (2015) How well do radiographic, clinical and self-reported diagnoses of knee osteoarthritis agree? Findings from the Hertfordshire cohort study. SpringerPlus 4:177. https://doi.org/10.1186/s40064-015-0949-z

17. Parsons C, Fuggle NR, Edwards MH, Goulston L, Litwic AE, Jagannath D, van der Pas S, Cooper C, Dennison EM (2017) Concordance between clinical and radiographic evaluations of knee osteoarthritis. Aging Clin Experiment Res 30:17-25. https://doi. org/10.1007/s40520-017-0847-z

18. Ferreira RM, Duarte JA, Goncalves RS (2018) Non-pharmacological and non-surgical interventions to manage patients with knee osteoarthritis: an umbrella review. Acta Reumatol Port 43(3): 182-200

19. Zhang W, Nuki G, Moskowitz RW, Abramson S, Altman RD, Arden NK, Bierma-Zeinstra S, Brandt KD, Croft P, Doherty M, Dougados M, Hochberg M, Hunter DJ, Kwoh K, Lohmander LS, Tugwell P (2010) OARSI recommendations for the management of hip and knee osteoarthritis: part III: changes in evidence following systematic cumulative update of research published through January 2009. Osteoarthr Cartil 18(4):476-499. https:// doi.org/10.1016/j.joca.2010.01.013

20. Nelson AE, Allen KD, Golightly YM, Goode AP, Jordan JM (2014) A systematic review of recommendations and guidelines for the management of osteoarthritis: the chronic osteoarthritis management initiative of the US bone and joint initiative. Semin Arthr Rheum 43(6):701-712. https://doi.org/10.1016/j.semarthrit .2013.11.012

21. Zhou Z, Hou Y, Lin J, Wang K, Liu Q (2018) Patients' views toward knee osteoarthritis exercise therapy and factors influencing 
adherence-a survey in China. Physician Sportsmed 46(2):221227. https://doi.org/10.1080/00913847.2018.1425595

22. Nicolson PJA, Hinman RS, French SD, Lonsdale C, Bennell KL (2018) Improving adherence to exercise: Do people with knee osteoarthritis and physical therapists agree on the behavioral approaches likely to succeed? Arthr Care Res 70(3):388-397. https://doi.org/10.1002/acr.23297

23. Oliver H, Jameson KA, Sayer AA, Cooper C, Dennison EM (2007) Growth in early life predicts bone strength in late adulthood: the Hertfordshire Cohort Study. Bone 41(3):400-405. https ://doi.org/10.1016/j.bone.2007.05.007

24. Egger P, Duggleby S, Hobbs R, Fall C, Cooper C (1996) Cigarette smoking and bone mineral density in the elderly. J Epidemiol Community Health 50(1):47-50
25. Cushnaghan J, Cooper C, Dieppe P, Kirwan J, McAlindon T, McCrae F (1990) Clinical assessment of osteoarthritis of the knee. Ann Rheum Dis 49(10):768-770

Publisher's Note Springer Nature remains neutral with regard to jurisdictional claims in published maps and institutional affiliations. 